\title{
Evolution of fit-for-purpose biomarker validations: an LBA perspective
}

\author{
Robert J Neely ${ }^{*}, 1$ \\ ${ }^{1}$ Senior Research Investigator, Bioanalytical Sciences - Biomarkers, Bristol-Myers Squibb, Route 206 \& Province Line Road, \\ Princeton, NJ 08543-4000, USA \\ *Author for correspondence: Tel.: +1 609252 6520; robert.neely@bms.com
}

Biomarker ligand-binding assays need to be validated for use on clinical studies in the drug development process. There is not one single guidance to cover all types of biomarker assays and their intended uses. Therefore, it is up to the scientist to piece together a validation strategy based on published papers and other sources. Shown here is a summary of what to take into consideration during a validation and how to apply it for use in the drug development process.

First draft submitted: 5 December 2017; Accepted for publication: 14 February 2018; Published online: 20 June 2018

Keywords: biomarker $\bullet$ biomarker validation $\bullet$ fit-for-purpose $\bullet$ intended use

\section{Piecing together the validation strategy}

Biomarkers can be widely used with a variety of intended purposes during drug development. These purposes can range from exploratory hypothesis generation, internal decision making, to clinical decisions or in vitro diagnostics (IVDs). Therefore, the biomarker assay and its measurements need to be suitable for the intended purpose and a one-size-fits-all approach for assay validation is not appropriate. IVDs and clinical decision-making assays are products that have cleared, or have been approved by, the US FDA for a very specific intended use. As such, they have very clear regulations centered on how to validate the assay and apply the results for clinical utility. However, biomarker assays for all other purposes were left in question. Adapting the PK method validation best practices was a good starting point, although it seemed insufficient to cover the spectrum of possible biomarker assays and their intended use. Consequently, this disparity led to the development of several white papers that described a fit-for-purpose approach implemented to help identify the best strategy for protein biomarker assay validations [1-3]. Addressing gaps not included in the FDA draft guidance, these papers elaborate on biomarker biology, the theory of relative accuracy as well as the issues surrounding endogenous biomarkers and appropriate quality control (QC) material. However, despite the recommendation of a 'continuous and evolving fit for purpose strategy', these white papers focused solely on de novo single analyte assays.

With the explosion of biomarker use during drug development and the expansion of personalized medicine, biomarker assays have become common in all phases of drug development. With the increased demand for more biomarker data, bioanalytical scientists had to rely on commercially available assays as well as multiplexed analysis to achieve the desired speed for the clinic. Building off of the previously published white papers, and the FDA guidance for bioanalytical method validation, new white papers were published to help address the analytical issues and gaps that could arise with commercial or multiplexed analysis $[4,5]$. This included a closer look at kit-to-kit and lot-to-lot variability of reagents for assays, as well as a higher understanding of cross-reactivity between calibrators, antibody reagents and the compromises to make when it comes to minimum required dilution and sensitivity needs. With the number of white papers expanding as fast as the intended uses for biomarkers, the industry on a whole was left wanting a revised strategy that could be applied for all biomarker assays. In order to address some of these concerns, several conference topics have focused on biomarker validations in order to obtain a consensus from the bioanalytical scientists and the FDA [6,7]. Not reaching a total consensus, it was clear that biomarker assays need to be defined by two principles; one, for their context of use, and two, for the biology surrounding the endogenous biomarker. By defining these two principles, the scientists can apply the analytical parameters that need to be addressed during validation of the assay. 
Ultimately, understanding the biology of the biomarker is one of the biggest hurdles when it comes to developing and validating the bioanalytical assay. Complications can arise from preanalytical factors such as variability within sample collection times, matrix and patient biology and background. Understanding the biological variability of the biomarker and the implications on assay data will help guide the appropriate validation strategy [8]. Another challenge with regard to relative accuracy is the calibrator material selection and the resulting calculations. This stems from the fact that there is no 'gold standard' for most protein biomarkers. This was highlighted by several white papers published focusing on the issues of biomarker calibrator material selection and characterization, as well as comparing the performance of the calibrator to its endogenous counterpart [9-11]. Without knowing the exact nature of the endogenous protein, or how it can change, the acceptance criteria and validation parameters for each assay remain to be determined by the scientists for each intended use. Given, there is a large variety of intended uses spanning the industry and drug development, as well as the fact that biomarkers can change their intended use during the course of the drug development process; means a continuum for a validation strategy should be adopted. The evolution of this strategy will build upon the previous guidance, the published white papers and need to incorporate aspects from the current literature such as calibrator material selection, parallelism, use of commercial kits and multiplexing.

\section{Applying the validation strategy}

The first step in applying a biomarker validation strategy is to recognize that this is not a PK assay and therefore should not start in the same manner as a PK assay validation. When the biomarker has been identified and its specific intended use declared, the primary concern is to identify all possible sources of a potential calibrator material. The more material to test in your assay, the better you will appreciate the limitations of the reagents being used. The secondary concern is to identify sources for high endogenous biomarker samples from relevant patient populations. Being able to match the calibrator material performance to the endogenous biomarker of interest will frame the interpretation of the assay data as well as the requisite validation strategy. Not all calibrator material will behave similar to the endogenous biomarker in matrix, or buffer. Thus, calibrators will need to be screened, as well as buffers, to investigate that relationship. As easy as this is to acknowledge in principle, executing this for each biomarker assay is virtually impossible and the extent will be defined by the intended use. This is an early issue in the discovery of the biomarker or very early in the drug development process when there is little time for in-depth investigation. Therefore, the responsibility is on the scientist to determine the amount of rigor necessary, or possible, for determination of the calibrator material and the performance based on the parallelism and dilution linearity experiments conducted for the intended use. Whether this is for a de novo assay, a commercial kit or a multiplex assay, this should always be performed to better understand what is being measured and the limitations with regard to reagents and relative accuracy.

Acceptable performance for each biomarker assay will vary, thus it is the judgement of the scientist to determine if the assay is fit for the intended use. Conducting the parallelism and dilution linearity experiments will give insight into the validation parameters for the minimum required dilution (MRD), range, sensitivity as well as QC sample selection. Agreement throughout industry indicates that biomarker assays should have at least one fully endogenous QC sample, if not more. Combining endogenous QCs with a mixture of spiked matrix, or buffer QCs, analytical performance can be assessed [1-3]. Determining analytical ranges, specificity, selectivity, stability and reproducibility using QCs will also guide in the proper selection of the acceptance criteria for the biomarker assay and its intended use. In using QC material, it is critical to understand the difference between endogenous biomarker and calibrator material stability. Both will need to be monitored during the validation to inform on the proper strategy for the continued use of the biomarker assay. Regardless of the number of QCs, QC material should be prepared in bulk to ensure the capability of bridging new lots of kits, or reagents, for the life cycle management of the assay. The number of experiments needed for the bridging will depend on the nature of the assay, whether it is a de novo assay, commercial kit or multiplexed $[4,5]$. If there are significant changes to either the components, kits, calibrators or antibodies, a re-validation will be required.

Outlined above is the starting point for biomarker assay validations and the basis for the continuum strategy. Identifying the biomarker, investigating the biological variability and background and defining an intended use for the data will dictate the validation parameters to be assessed. The most important parameters to investigate are parallelism, MRD and the basic analytical performance characteristics of the assay (LOD, LLOQ, ULOQ, precision, reproducibility, specificity, selectivity and stability). The level and extent of investigation of these parameters will be based on the intended use of the data and the scientific judgement of the bioanalytical scientists. As mentioned 
above, judgement is based on the published white papers, conferences and FDA draft guidance. However, these are not the only sources to be utilized. As the biomarker data starts to be used for clinical decision-making purposes, the scientists will need to increase the level of investigation and validation by incorporating principles laid out in the recent draft document prepared by the Critical Path Institute (C-Path) [12]. Ultimately, as the biomarker use evolves, the scientists will need to combine the analytical validation described above with the clinical validation as described in the BEST resource [13] and the draft document being prepared by the C-Path institute to form the continuum strategy.

\section{Financial \& competing interests disclosure}

The author has no relevant affiliations or financial involvement with any organization or entity with a financial interest in or financial conflict with the subject matter or materials discussed in the manuscript. This includes employment, consultancies, honoraria, stock ownership or options, expert testimony, grants or patents received or pending, or royalties.

No writing assistance was utilized in the production of this manuscript.

\section{Open access}

This work is licensed under the Attribution-NonCommercial-NoDerivatives 4.0 Unported License. To view a copy of this license, visit http://creativecommons.org/licenses/by-nc-nd/4.0/

\section{References}

1. Lee JW, Devanarayan V, Barrett YC et al. Fit-for-purpose method development and validation for successful biomarker measurement. Pharm. Res. 23(2), 312-28 (2006).

2. Hougton R, Gouty D, Allinson J et al. Recommendations on biomarker bioanalytical method validation by GCC. Bioanalysis 4(20), 2439-2446 (2012).

3. US FDA. Bioanalytical method validation, draft guidance for industry. In: CDER and CVM. MD, USA (2013).

4. Jani D, Allison J, Berisha F et al. Recommendations for use and fit-for-purpose validation of biomarker multiplex ligand binding assays in drug development. AAPS J. 18(1), 1-14 (2015).

5. Khan MU, Bowsher RR, Cameron $\mathrm{M}$ et al. Recommendations for adaptation and validation of commercial kits for biomarker quantification in drug development. Bioanalysis 7(2), 229-242 (2015).

6. Arnold ME, Booth B, King L et al. Workshop Report: Crystal City VI - Bioanalytical Method Validation for Biomarkers. AAPS J. 18(6), 1366-1372 (2016).

7. Richards S, Amaravadi L, Pillutla R et al. 2016 White Paper on recent issues in bioanalysis: focus on biomarker assay validation (BAV): (Part 3 - LBA, biomarkers and immunogenicity). Bioanalysis 8(23), 2475-2496 (2016).

8. Dakappagari N, Zhang H, Stephen L, Amaravadi L, Khan M. Recommendations for clinical biomarker specimen preservation and stability assessments. Bioanalysis 9(8), 643-653 (2017).

9. Stevenson LF, Purushothama S. Parallelism: considerations for the development, validation and implementation of PK and biomarker ligand-binding assays. Bioanalysis 6(2), 185-198 (2014).

10. King LE. Parallelism experiments in biomarker ligand-binding assays to assess immunological similarity. Bioanalysis 8(23), 2387-2391 (2016).

11. Cowan K, Amaravadi L, Cameron M. Recommendations for selection and characterization of protein biomarker assay calibrator material. AAPS J. 19(6), 1550-1563 (2017).

12. Piccoli SP, Sauer JM,Amur SBiomarker Assay Collaborative Evidentiary Considerations Writing Group. et al.; Points to Consider Document: scientific and regulatory considerations for the analytical validation of assays used in the qualification of biomarkers in biological matrices. https://healthpolicy.duke.edu/sites/default/files/atoms/files/white_paper_6_14_17_0.pdf

13. BEST (Biomarkers, EndpointS, and other Tools) Resource Glossary. FDA-NIH Biomarker Working Group, MD, USA (2016). 
